\title{
Rückwärts schauend in die Zukunft: Utopien des Alten Orients
}

\author{
Stefan M. Maul \\ HEIDELBERG
}

Nachdem Schamschi-Adad I. im frühen 2. Jt. v. Chr. dem Haupttempel der Stadt Assur seine monumentale Gestalt verliehen hatte, blieb der Grundplan des Gebäudes über mehr als ein ganzes Jahrtausend hinweg weitgehend unverändert. ${ }^{1}$ Obgleich das Gotteshaus immer wieder renoviert und nach einer Brandkatastrophe sogar vollständig neu errichtet werden mußte, versuchte keiner der mächtigen mittelund neuassyrischen Könige - all den stürmischen politischen und gesellschaftlichen Veränderungen zum Trotz-dessen Struktur grundlegend zu verändern.

Doch im frühen 7. Jh.v. Chr. faßte König Sanherib einen bis dahin unvorstellbaren Beschluß: Er ließ den großen Vorhof des Tempels meterhoch aufschütten und so die dort befindlichen Wasserbecken und das dem Tempel vorgelagerte, mit Schmelzfarbenbildern geschmückte Podium mitsamt seinen Treppen und Rampen in dem Füllschutt einfach verschwinden. Ein gewaltiger Anbau wurde errichtet, der nicht nur für ein ganz ungewohntes äußeres Erscheinungsbild des AssurTempels sorgte, sondern wohl auch den altehrwürdigen Kultbetrieb in völlig neue Formen zwang. ${ }^{2}$ Auch ließ Sanherib vor den Toren der Stadt ein in Gartenanlagen eingebettetes Festhaus errichten, das-babylonischen Bräuchen folgend-das Ziel einer feierlichen Neujahrsfestprozession sein sollte, welche ihren Weg heraus aus der Stadt in die im Frühjahr auflebende Natur nahm. ${ }^{3}$

Der König, dessen mannigfachen Versuche, Babyloniens Eigenständigkeit mit friedlichen und mit kriegerischen Mitteln zu unterbinden, allesamt gescheitert waren, hatte 689 v. Chr. in blankem $\mathrm{Haß}$ die belagerte und eingenommene Stadt Babylon von den aufgestauten Fluten des Euphrat regelrecht hinwegschwemmen lassen und hoffte so, für alle Zeiten den Gegner ausgelöscht zu haben. Das altehrwürdige kultische und religiöse Erbe Babylons sollte von nun an in Assur aufgehen und die ausgelöschte Kulttopographie Babylons für alle Zukunft in dem umgestalteten Assur weiterleben.

Die Umbauten des Assur-Tempels mögen bei der Priesterschaft Empörung ausgelöst haben. Freilich versuchte Sanherib jegliche Kritik im Keime zu ersticken, indem er behauptete, in Wahrheit mit seinen Reformen der mušaklil paraș Ešarra

1. Zum Assur-Tempel siehe Haller, Andrae 1955, 6 ff.; Andrae 1977, 46-49; 121-129; 165-170; 224-226; Heinrich 1982, 198-199; 232-233; 273-275.

2. Siehe Frahm 1997, 286.

3. Siehe Haller, Andrae 1955, 74-80; Andrae 1977, 219-224; Heinrich 1982, 275-277. 
mašûti ${ }^{4}$ zu sein-derjenige, der nach langer Zeit "die in Vergessenheit geratenen Kultordnungen" des Assur-Tempels erst wiederhergestellt habe. Auch das neu errichtete Neujahrsfesthaus, so Sanherib, sei von ihm endlich wieder an seinem Platz außerhalb der Stadt errichtet worden, wie es "seit ewiger Zeit" Sitte gewesen, aber schändlicherweise aufgrund von "Irrungen und Wirrungen" ${ }^{5}$ schon lange aufgegeben worden sei.

Wir wissen nicht, welche Argumente Sanherib und seine Berater bemühten, um eine solche Behauptung zu stützen. Hinweise auf Vorgängerbauten konnten die archäologischen Untersuchungen in Assur jedenfalls nicht erbringen. Aber ganz unabhängig von der Frage, ob hier tatsächlich eine uralte Kulttopographie wiederhergestellt wurde, oder ob wir es mit einem dreisten making of tradition ${ }^{6} \mathrm{zu}$ tun haben, kann man an der Sanherib-Inschrift ermessen, wie gewaltig hoch die Autorität des Althergebrachten im Assyrien des Sanherib gewesen sein muß.

In der Tat ist das Interesse der mesopotamischen Kultur an der eigenen Vergangenheit über viele Jahrhunderte hin auf Schritt und Tritt allgegenwärtig. In den Hinterlassenschaften der assyrischen und der babylonischen Kultur des zweiten und ersten vorchristlichen Jahrtausends läßt sich die mächtige, alles durchdringende normative Kraft des Alt(hergebracht)en schon auf den ersten Blick erkennen. Exemplarisch wollen wir dem in einigen Beispielen nachgehen, indem wir einen Blick auf die Sprachen, die Schrift und die materiellen Hinterlassenschaften des Alten Orients werfen.

\section{Die Sprachen des Alten Orients}

Die zahlreichen Inschriften der Herrscher Assyriens und Babyloniens, die man in den Fundamenten von Tempeln und Palästen für die Nachwelt hinterlegte oder sichtbar auf Reliefs und Stelen anbrachte, wurden in einer Kunstsprache verfaßt, die-weit entfernt von der zeitgenössischen semitischen Sprache des Alltagssich an der altertümlichen, als klassisch empfundenen babylonischen Sprache orientierte, die zu Beginn des 2. Jahrtausends v. Chr. gesprochen wurde und schon damals mannigfache Archaizismen aufwies. ${ }^{7}$ Auch die übrige Literatur (religiöse und gelehrte Texte, Epen und Mythen) bediente sich dieser, von uns "StandardBabylonisch" genannten gehobenen Sprachform. Mit ihrem altertümlichen Klang beschwor sie nicht nur die altehrwürdige Zeit des Königs Hammurapi, der im 18. Jh. v. Chr. ganz Mesopotamien und Teile Syriens zu einem mächtigen Reich geeint hatte. Das "Standard-Babylonische" brachte auch diejenige Sprachform des Babylonischen immer wieder zum Klingen, in der im frühen 2. Jahrtausend v. Chr. erstmals in einer semitischen Sprache im größeren Umfang Literatur schriftlich niedergelegt worden war.

Das Sumerische, das älteste, mit keiner bekannten Sprache verwandte Idiom des Zweistromlandes, galt noch um die Zeitenwende-zweitausend Jahre nachdem es als gesprochene Sprache aufgehört hatte zu existieren-als die ehrwürdigste Sprache. Sumerische Lieder, Hymnen und Gebete, die bereits im 3. Jahrtausend v. Chr. entstanden, wurden immer wieder abgeschrieben und mit babylonischen

\footnotetext{
4. Schroeder 1922, Text Nr. 122:3 f.

5. Schroeder 1922, Text Nr. 122:26 (ultu ūmẽ rūqūti; ešâti u sahmašāti).

6. Siehe Frahm 1997, 286.

7. Siehe von Soden 1932/33; ders. 1995, 3 \& 2d; Groneberg 1972.
} 
Übersetzungen versehen. Sie nahmen gemeinsam mit späteren Nachschöpfungen aus dem 2. und 1. Jahrtausend v. Chr. bis zum Ende der Keilschriftkultur um das 2. Jh. unserer Zeit eine wichtige Stellung im Götterkult ein. Darüber hinaus lebte das Sumerische, ganz ähnlich wie das Lateinische in unserer Kultur, als Gelehrtensprache fort. So wie die Humanisten der frühen Neuzeit ihre Namen latinisierten, übertrugen babylonische und assyrische Gelehrte ihre semitischen Namen gerne ins Sumerische und verfassten gelehrte Texte in diesem Idiom. Vor allem in den großen Städten, die als die Zentren der frühen sumerischen Kultur galten, ließen assyrische und babylonische Könige auch noch im 1. Jahrtausend v. Chr. ihre Bau- und Weihinschriften den alten Traditionen folgend in sumerischer Sprache verfassen.

\section{Schrift}

Königsinschriften des ersten Jahrtausends v. Chr. wurden darüber hinaus nicht selten mit altertümlichen Keilschriftzeichenformen niedergeschrieben, die im zeitgenössischen Alltagsleben schon viele Jahrhunderte außer Gebrauch waren und von weniger Gebildeten ganz sicher nicht entziffert werden konnten. ${ }^{8}$ Die Verwendung uralter Zeichenformen ist freilich kein Phänomen, das sich auf das erste vorchristliche Jahrtausend beschränkt. Bereits Hammurapi von Babylon, dessen Zeit man später als "klassisch" betrachten sollte, ließ den Text seiner berühmten Gesetzesstele in einem Schriftduktus niederschreiben, der einen über 600 Jahre älteren paläographischen Entwicklungsstand der Keilschrift widerspiegelt und damit bewußt an die Zeit der altakkadischen Könige anknüpft, die im 24. Jh. v. Chr. erstmals eine Einigung der mesopotamischen Stadtstaaten erzwangen. Dem Vorbild Hammurapis folgend ließ noch Nebukadnezar II. im 6. Jh. v. Chr. seine Inschriften bei dem prächtigen Ausbau von Babylon mit den feierlich wirkenden Zeichen schreiben. Die Inschriften seiner Epoche ahmen nicht nur die Sprache der Hammurapi-Zeit nach, sondern bedienen sich oft auch der alten, im Alltag längst außer Gebrauch gekommenen orthographischen Konventionen. ${ }^{9}$

Die gelehrten Schreiber des 2. und 1. Jt. v. Chr. legten-modernen Assyriologen gleich-paläographische Zeichenlisten an ${ }^{10}$ studierten alte Texte und fertigten von ihnen Tontafelfaksimiles, die so gelungen erscheinen, daß sich bisweilen auch Assyriologen über das wahre Alter dieser Dokumente täuschen lassen. So konnte sich der neuassyrische König Assurbanipal (669-631 v. Chr.) sogar rühmen, er habe Inschriften "aus der Zeit vor der Sintflut" entziffern können.

8. Siehe beispielsweise die Tontafel-Inschrift des Asarhaddon K 7943 (Borger 1956, $117 \S 90$ und Taf. 5), die in der sehr altertümlichen Linearschrift des 3. Jt. v. Chr. verfaßt wurde, und den Tonzylinder des Assurbanipal BM 86918 (CT 9 Nr. 6-7). Siehe ferner auch Maul 2012 a und 2012 b.

9. Dazu zusammenfassend Schaudig $2001,86-88$ und 315 .

10. Stellvertretend sei hier auf in Ninive, Kalbu (Nimrud) und Assur gefundene Zeichenlisten verwiesen, in denen einem Keilschriftzeichen jeweils ein Bildzeichen gegenübergestellt ist, das zumindest nach Ansicht der Kompilatoren am Anfang der Schriftentwicklung stand (z. B. Wiseman, Black 1996, Text Nr. 229; siehe Marzahn, Schauerte 2008, 434 Abb. 315 mit weiteren Beispielen). Das Tafelfragment Wiseman, Black 1996, Text Nr. 235 stellt ferner unter Beweis, daß auch mit diesen erlernten uralten Bildzeichen neue Texte geschrieben wurden.

11. Streck 1916, 256, Tontafelinschrift L4, Vs. 18. 


\section{Materielle Hinterlassenschaften}

Das Interesse an einer als "klassisch" empfundenen Vergangenheit manifestierte sich in Mesopotamien jedoch nicht nur in der Verwendung von altertümlicher Sprache und Schrift. Auch in der materiellen Kultur Mesopotamiens lassen sich immer wieder Rückgriffe auf weit zurückliegende Perioden nachweisen, denen hier freilich nicht im einzelnen nachgegangen werden kann. Ein eindrucksvolles Beispiel soll genügen. Überraschend erscheint dem modernen Leser die in neubabylonischen Königsinschriften aus dem 6 . vorchristlichen Jahrhundert nicht selten anzutreffende Schilderung, daß im Auftrag des Herrschers in seit Jahrtausenden bestehenden Tempelbezirken regelrechte großflächig angelegte archäologische Ausgrabungen unternommen wurden, um Reste von uralten, manchmal längst vergessenen Kulteinrichtungen ausfindig zu machen. ${ }^{12}$ In einer Inschrift berichtet der babylonische König Nabonid:

Die Fundamentsteine des Tempels E’ulmasch zu Akkade, die seit der Zeit des Sargon, des Königs von Babylon, und des Naram-Sin, seines Sohnes, Königen früherer Zeit, und gar bis zur Regierungszeit des Nabonid, des Königs von Babylon, niemandem zu Gesicht gekommen waren, hatte Kurigalzu, der König von Babylon, ein König früherer Zeit, gesucht, aber die Fundamentsteine von E'ulmasch fand er nicht (. . .). Nebukadnezar, der König von Babylon (. . .), entsandte seine Arbeitstruppen in großer Zahl, um nach jenen Fundamentsteinen des E'ulmasch zu suchen, er mühte sich ab, grub in die Tiefe, aber die Fundamentsteine von E'ulmasch fand er nicht. Ich dagegen, Nabonid, der König von Babylon, erschaute während meiner rechtmäßigen Regierung in der Furcht vor Ischtar von Akkade, meiner Herrin, einen Opferschaubefund. Schamasch und Adad antworteten mir mit einem zuverlässigen Jawort; daß ich finden sollte die Fundamentsteine jenes E'ulmasch, legten sie mir als günstigen Fleischbefund in meinen Opferschaubescheid. Meine Mannen in großer Zahl entsandte ich, zu suchen nach den Fundamentsteinen jenes E’ulmasch. Drei Jahre lang durchgrub ich die Senkschächte des Nebukadnezar, des Königs von Babylon. Rechts und links, vorwärts und rückwärts suchte ich, aber ich fand sie nicht. Da sprachen sie zu mir also: "Nach jenen Fundamentsteinen haben wir gesucht, aber wir haben sie nicht zu Gesicht bekommen. ${ }^{13}$

Erst in einem späteren Anlauf bekommen die Reste des um 2350 v. Chr. errichteten sargonischen Baus gefunden, und Nabonid konnte dann, wie er an anderer Stelle formulieren ließ, "keinen Finger breit zu weit vorspringend oder zurücktretend über den alten Fundamentsteinen"14 das neue Fundament des Tempels anlegen.

Das Ziel derartiger Ausgrabungen bestand darin, den ältesten Bauzustand eines Tempels zu ermitteln. So lange wurden die Reste der jüngeren Bauphasen abgetragen, bis man glaubte, auf die früheste in den Fundamenten dokumentierte Gestalt des Gotteshauses gestoßen zu sein. Das archäologische Interesse babylonischer (und auch assyrischer) Könige an der Tempelarchitektur ihres "Altertums" war freilich nicht in erster Linie antiquarischer Natur. Vielmehr wurde der ermittelte "antike" Bauplan benötigt, um den Tempel in seiner ursprünglichen, von den Veränderungen der Zeiten unverfälschten Gestalt wiederherzustellen und beim

12. Siehe Goosens 1948.

13. Nach Schaudig 2001, 454-456 und 463-464, Exemplar 2 II 28-34, 45-63.

14. Siehe z. B. Schaudig 2001, 452, Exemplar 2 II 8. 
Neubau des Gotteshauses "keinen Finger zuviel und keinen Finger zuwenig" von dem alten und uranfänglichen Plan abzuweichen. Bezeichnenderweise bedeutet die akkadische (und auch die sumerische) Wendung ${ }^{16}$, die in den Wörterbüchern mit "wiederherstellen" oder "restaurieren" wiedergeben wird, wörtlich übersetzt "(eine Sache) an den jeweils für sie vorgesehenen / an den ihr gebührenden Platz zurückführen.” Dahinter ist deutlich die mesopotamische Vorstellung zu spüren, daß allen Dingen in der Welt jeweils ein fester, ewiger und unverrückbarer Platz zugewiesen ist. Diesen von den Zeitläuften immer wieder in Frage gestellten Platz galt es, mit der Rekonstruktion des alten Tempels wiederzugewinnen.

Mythen, die sich um die Tempel Babyloniens ranken, wissen $\mathrm{zu}$ berichten, daß diese Gotteshäuser keineswegs von Menschenhand, sondern als Teil des Schöpfungswerkes zum Anbeginn der Zeiten von den Göttern selbst errichtet worden seien. ${ }^{17}$ Die Wiederherstellung des Tempels nach dem unverfälschten göttlichen Plan sollte den königlichen Bauherrn, die Menschen und das Staatswesen wieder in den ungetrübten, segensreichen Uranfang zurückversetzen.

Die Suche der Babylonier und Assyrer nach ihrem "Altertum" entpuppt sich somit als das Streben nach der klaren uranfänglichen Ordnung, der die Götter selbst im Schöpfungsakt ihre Gestalt gegeben hatten. Bezeichnenderweise wird diese von Alterungsprozessen noch unbeschadete Urzeit stets mit dem Begriff der "fernen Zeit" verbunden, der so wie die ferne Vergangenheit auch die ferne Zukunft meinen kann; gleichsam so, als könnten die ferne Vergangenheit und die ferne Zukunft auf einer gebogenen Zeitachse zueinander finden und einander durchdringen.

Ein Blick in die mythischen Texte Mesopotamiens zeigt sehr rasch, daßtatsächlich auch sämtliche kulturellen Errungenschaften, sei es die Baukunst, die Kunst der Schreiber, Goldschmiede, Schreiner usw., zu Offenbarungen des (Weisheits-)Gottes Ea erklärt wurden, die dieser der Menschheit zum Anbeginn der Zeiten in die Hand gegeben hatte. Noch Berossos, ein Marduk-Priester des 3. Jh. v. Chr., der mit seinem griechischsprachigen Werk Babyloniaka ${ }^{18}$ der hellenistischen Welt Geschichte und Kultur des alten Babyloniens nahebrachte, hielt dieses Selbstverständnis der babylonischen Kultur für wesentlich: Ein fischgestaltiges Wesen namens Oannes ${ }^{19}$ sei, so Berossos, im ersten Jahr der Welt, also unmittelbar nach Erschaffung von Himmel, Erde und Menschen, aus dem Meer gestiegen und habe "die Menschen die Schriftkunde und die mannigfaltigen Verfahrungsweisen der Künste, die Bildungen von Städten und die Gründungen von Tempeln (gelehrt) ... was nur immer der Häuslichkeit des Lebens der Welt zustattenkommt, überlieferte es den Menschen; und seit jener Zeit", so Berossos, "werde von keinem anderen mehr auch nur irgendetwas erfunden".

Auch die Könige Babyloniens und Assyriens suchten, obgleich sie sich in ihren Inschriften stolz auf ihre Vorgänger beriefen, die Jahrtausende vor ihnen das Land regiert, geeint und zu Machtfülle gebracht hatten, die Zeiträume, die sich zwischen diese und den Uranfang der Schöpfung gelegt hatten, zu überwinden. Das

15. Lambert 1968/69, 5 Zeile 24 (Inschrift des Nabonid; weitere Belege AHw 1399a).

16. ki-bi-šè gi ${ }_{4}$ (sumerisch) = ana ašrǐšu turru (akkadisch).

17. Siehe z. B. van Dijk 1998.

18. Siehe Schnabel 1923 und Burstein 1978.

19. Zu Oannes $\left(=\mathrm{u}_{4}\right.$-an, $\mathrm{u}_{4}{ }^{-\mathrm{d}} \mathrm{an}, \mathrm{u}_{4}$-an-na; $\left.u_{4}-m a-{ }^{\mathrm{d}} a-n i m, u^{-\mathrm{d}} a-n i m\right)$ in der keilschriftlichen Literatur siehe Lambert 1962, 74; Hallo 1963, 176 Anm. 7; Borger 1974, 186 und George 1992, 269.

20. Schnabel 1923, 253. 
jährlich durchgeführte Neujahrsfest ist ein beredtes Zeugnis dieses Bestrebens. In diesem bedeutsamen Staatsritual präsentierte sich der König Hand in Hand mit dem Weltengott (d.h. mit dem Kultbild des Gottes), um mit diesem den uranfänglichen Kampf des Gottes gegen die Mächte des Chaos, den Sieg über diese Widersacher der Ordnung und die sich anschließende Erschaffung der Welt im Kultgeschehen zu reaktualisieren. Mesopotamische Herrscher legitimierten sich nicht nur dadurch, daß sie "von ewigem Samen" ${ }^{21}$, von "kostbarem Samen aus der Zeit vor der Sintflut" ${ }^{22}$ und aus "Familien der Urzeit" ${ }^{23}$ stammten. Auch einem aus neubabylonischer Zeit bekannten Mythos zufolge schufen die Götter "den König" sogleich nach der Erschaffung der Menschen, damit er diese "recht leite". ${ }^{24}$ Die gottgewollte Aufgabe eines Königs bestand demnach darin, die in der Schöpfung gewordene geordnete Welt zu bewahren, zu verteidigen und wieder in den Zustand zurückzuversetzen, aus dem heraus sie sich deformiert hatte. Das Idealbild der Gesellschaft und des Staatswesens, die Utopie der Mesopotamier, war somit stets in der Urvergangenheit und nie in der Zukunft angesiedelt. Daher erstaunt es nicht, daß im ersten vorchristlichen Jahrtausend assyrische Könige ihre Kriegszüge gegen die Feinde des Reiches durch subtile Anspielungen als den sich in den Zeiten immer wiederholenden Urkampf des Weltengottes gegen die Mächte des Chaos schilderten, der doch endlich mit dem triumphalen Sieg und der Ordnung der Welt im Schöpfungswerk endete. ${ }^{25}$ So bildete der Mythos den Rahmen, in dem Geschichte immer wieder auskristallisiert.

Der stete Blick auf das Uranfängliche, das Ehren und immer wieder neu Entdecken des Alten ist freilich nur auf den ersten Blick, nur äußerlich rückwärtsgewandt. Der enorme Aufwand, mit dem im Rahmen von divinatorischen Verfahren altorientalische Fürsten durch die Zeiten hinweg mit ihren Beratern Pläne und Absichten auf deren Zukunftsfähigkeit hin prüften, spricht eine ganz andere Sprache. Umfangreiche Staatsarchive aus verschiedenen Epochen des Alten Orients zeigen uns nämlich, daß Könige und Regierungen einer bewußten Zukunftsgestaltung höchste Priorität einräumten.

Auch aus unserer Sicht kann die altorientalische Kultur des Zweistromlandes keineswegs als eine Kultur beschrieben werden, die krampfhaft am Altbewährten festhielt und Experimentieren, Weiterentwicklung und Fortschritt nicht zuließ oder à la longue behinderte.

Geopolitische, ökonomische und nicht zuletzt auch ökologische Bedingungen erzwangen in der bewegten altorientalischen Geschichte immer wieder neue Herrschaftsformen. Sie führen vom Priesterfürstentum der kleinflächigen frühen sumerischen Stadtstaaten des 3. vorchristlichen Jahrtausends bis hin zu dem Großkönigtum des assyrischen Reiches, das ein Gebiet vom Zagros bis einschließlich Ägypten, von Anatolien bis tief in die arabische Halbinsel zu kontrollieren hatte. Immer wieder mußten neue, den veränderten Bedingungen angepaßte Formen der Verwaltung, neue Technologien, nicht zuletzt auch der Situation angemessene Mittel

21. Siehe CAD Z 95 f. s.v. zēru 4b.

22. Siehe Frame 1995, 25, Nebuchadnezzar I B.2.4.8, Zeile 8: zēru nașru ša lām abūbi.

23. Der assyrische König Asarhaddon (680-669 v. Chr.) bezeichnete sich und die assyrische Königsdynastie als zēr šarrūti kisitti ŝati, "Same des Königtums, Stammbaum der Ewigkeit" (siehe Borger 1956, 32, Brs. A., Zeile 17 [dort übersetzt als: "königlicher Same, Uradliger"]).

24. Mayer 1987.

25. Hierzu siehe Maul 1999, 211. 
von Politik und Kriegsführung entwickelt werden. Im mittel- und neuassyrischen Reich etwa entstand ein ausgeklügeltes System der Provinzverwaltung, auf das die späteren Achämenidenkönige ebenso zurückgreifen konnten wie auf das von den assyrischen Großkönigen ausgebaute Netz der Königsstraßen.

Mesopotamien wußte sich auch neue Technologien, sofern sie Vorteile versprachen, rasch nutzbar zu machen. Von hier aus nahm der neuartige Werkstoff Bronze ebenso wie später auch das Eisen seinen Siegeszug, um Landwirtschaft, Kriegswesen und zahlreiche andere Bereiche des Lebens erheblich effektiver zu gestalten. Auch etwa das Glas ist wohl mesopotamischer Experimentierfreude zu verdanken.

Das Streben, langfristig über das Netz kausaler Zusammenhänge in der Welt genaueren Aufschluß zu erlangen, führte zu einem enormen, beweglichen Forschergeist. In den Tempeln Babyloniens wurde vom 8. Jh. v. Chr. an wohl lückenlos über Jahrhunderte hinweg in Form von Jahresberichten nicht nur über die Bewegung der Himmelskörper und das Wetter Rechenschaft abgelegt, sondern auch über die Preisentwicklung bestimmter ökonomisch wichtiger Güter, über Wasserstände und bemerkenswerte, als ominös eingestufte terrestrische Vorkommnisse, um Koinzidenzen mit den ebenfalls sorgsam verbuchten einschneidenden zeitgeschichtlichen Ereignissen zu erfassen. ${ }^{26}$ Ziel war es, Gesetzmäßigkeiten im Weltgeschehen zu ermitteln, um diese Erkenntnisse für politisches Handeln nutzbar zu machen. Im Dienste dieses Strebens entstand im Babylonien des ersten vorchristlichen Jahrtausends die rechnende Astronomie, ein Zweig babylonischer Wissenschaft, der bis heute fortbesteht.

Kurz, in eben dem Maße, in dem wir die mesopotamische Kultur als auf das Vergangene schauend beschreiben, können wir sie auch als eine Kultur sehen, die offen für Weiterentwicklungen und Neuerungen war.

Dennoch suchen wir Begriffe für "Fortschritt", "Entwicklung", "Weiterentwicklung" und "Neuerung" vergeblich im Wortbestand der assyrischen und der babylonischen Sprache. Allein ein Wort für "verändern, abändern" ist anzutreffen (nukkuru), allerdings mit einer unguten Konnotation. Denn von der gleichen Wurzel sind auch die Wörter gebildet, die "fremd", "feindlich", "Feind" (nakru) und "Feindschaft" (nukurtu) bedeuten. Freilich ist auch der Begriff des "Alten" nicht ausschließlich positiv besetzt. labīru, das "Alte", meint nämlich nicht allein das Altehrwürdige, sondern kann auch für das altersschwach und brüchig Gewordene stehen, das nach Erneuerung verlangt. Das Ersetzen des brüchig Gewordenen durch etwas Neues ist in der Begrifflichkeit der Babylonier dennoch nicht Fortschritt oder Neuerung genannt, sondern ana ašrīšu turru, wörtlich das "Zurückführen der Sache, an den ihr (seit alters her) gebührenden Platz". Reformen wurden dementsprechend in Mesopotamien, wie sehr auch immer sie mit dem zuvor Gewesenen brachen, grundsätzlich als das Wiederherstellen einer (im Laufe der Zeit brüchig gewordenen) Ordnung beschrieben. Die enormen, für die Menschheitsgeschichte prägenden Entwicklungen, die die Gesellschaft Mesopotamiens im Laufe von Jahrhunderten und Jahrtausenden durchmachte, wurde somit nicht als "Fortschritt" begriffen, sondern als Re-stauration, als "Rückschritt" hin zu einer uranfänglichen Ordnung und Stabilität. 
Im Gewand des Althergebrachten präsentierte sich so das Neue. Dies mag uns fremd erscheinen. Nur derjenige, der gewillt ist, sich auf diese mesopotamische Sicht der Dinge einzulassen, wird die darin verborgene Klugheit erkennen können. Denn Neues kann hier nicht ohne weiteres unverbunden in das Bestehende und das Gewesene implantiert werden. Es muß sich in wie immer gearteter Weise mit dem Alten auseinandersetzen, an das Alte anschließen, sich plausibel mit dem Alten verbinden. Angesichts der massiven Verwerfungen und Umstürze, der Kriege und Brüche, die die Geschichte Mesopotamiens prägen, erscheint dies als ein äußerst sinnvolles Mittel, die Gewalt des Neuen zu domestizieren, das Neue einer Prüfung zu unterziehen, am Bestehenden zu messen und ein Aushandeln neuer Strukturen zu erzwingen.

Es ist offensichtlich, daß die wichtige Frage was denn "alt» am Neuen war, Teil eines solchen Prozesses des Aushandelns gewesen sein muß und auf ganz verschiedene Weise beantwortet werden konnte. Die hermeneutische Oberhoheit über dieses gewichtige, mit Tradition und Geschichte eng verbundene Problem dürfte zunächst vorwiegend bei den Tempeln und ihren Priestern, mit zunehmender Komplexität der staatlichen Gebilde aber immer mehr bei den mächtigen Königen und ihren gelehrten Beratern gelegen haben.

Gelang es, das Neue als konstitutiven, zumindest in ovo angelegten Teil der eigenen Geschichte und Tradition plausibel zu machen, konnte das Neue als das Ureigene wahrgenommen werden und dadurch den Charakter des Bedrohlichen verlieren. Auch Errungenschaften die keineswegs mesopotamischen Ursprungs waren, konnten, nahmen sie nur das Gesicht des mesopotamisch-Althergebrachten an, problemlos in den als konstitutiv empfundenen Bestand mesopotamischen Kulturguts überführt werden. Die Formensprache des Alten, die als das Eigene empfunden wurde, ermöglichte so auch, Fremdes und Neues zu übernehmen, ohne daß es schmerzlich als Fremdes und Neues empfunden werden mußte. Nicht zuletzt diesem Mechanismus ist es geschuldet, daß es der mesopotamischen Kultur über Jahrhunderte immer wieder gelang, sich zu reformieren und eine Globalkultur mit mesopotamischem Antlitz hervorzubringen.

Erst als Babylon im Jahre 539 v. Chr. in die Hände der persischen Achämeniden fiel und Mesopotamien damit ein für alle mal die politische Oberhoheit über den Vorderen Orient verlor, zerbrach die normative Kraft mesopotamischer Traditionen.

Das Babylonische hörte dann auf, die lingua franca des Vorderen Orients zu sein. Das Aramäische und später das Griechische traten an seine Stelle. Auch Keilschrift und Tontafel verloren rasch an übernationaler Bedeutung. Die Formensprache der uralten mesopotamischen Kultur, der sozusagen die agency entglitten war, war nicht länger mehr geeignet, so wie in den vielen Jahrhunderten zuvor den Rahmen, das Gefäß für Neuerungen zu liefern. Von außen betrachtet mußte diese alte Kultur nunmehr nicht nur als im Alten verhaftet angesehen werden, sondern zunehmend auch als unzeitgemäß und veraltet.

\section{References}

Andrae, W.

1977 Das wiedererstandene Assur. Hrsg. von Barthel Hrouda. 2., durchges. u. erw. Aufl. München: Beck. 
Borger, R.

1956 Die Inschriften Asarhaddons Königs von Assyrien. Archiv für Orientforschung Beiheft 9. Graz: Selbstverlag des Herausgebers.

1974 Die Beschwörungsserie bīt mēseri und die Himmelfahrt Henochs. JNES 33: 183-196.

Burstein, S. M.

1978 The Babyloniaca of Berossus. Sources from the Ancient Near East 1/5, Malibu: Undena Publ.

Dijk, J. J. A. van

1998 Inanna raubt den "großen Himmel". Ein Mythos. Pp. 9-38 in Festschrift für Rykle Borger zu seinem 65. Geburtstag am 24. Mai 1994: tikip santakki mala bašmu. Cuneiform Mono-

Frahm, E. graphs 10. Edited by S. M. Maul. Groningen: Styx Publ.

1997 Einleitung in die Sanherib-Inschriften. Archiv für Orientforschung Beiheft 26. Wien: Institut für Orientalistik der Universität Wien.

Frame, G.

1995 Rulers of Babylonia. From the Second Dynasty of Isin to the End of Assyrian Domination (1157-612 BC). The Royal Inscriptions of Mesopotamia, Babylonian Periods Vol. 2. Toronto: University of Toronto Press.

George, A. R.

1992 Babylonian Topographical Texts. Leuven: Dep. Oriëntalistiek.

Goosens, G.

1948 Les recherches historiques à l"époque néo-babylonienne. RA 42: 149-159.

Groneberg, B.

1972 Untersuchungen zum hymnisch-epischen Dialekt der altbabylonischen literarischen Texte. Münster: Ph.D dissertation.

Haller, A. and Andrae, W.

1955 Die Heiligtümer des Gottes Assur und der Sin-Šamaš-Tempel in Assur. Wissenschaftliche Veröffentlichungen der Deutschen Orient-Gesellschaft 67. Berlin: Mann.

Hallo, W. W.

1963 On the Antiquity of Sumerian Literature. JAOS 83: 167-176.

Heinrich, E.

1982 Die Tempel und Heiligtümer im alten Mesopotamien. 1. Text. Berlin: de Gruyter.

Lambert, W. G.

1962 A Catalogue of Texts and Authors. JCS 16: 59-77.

1968/69 A New Source for the Reign of Nabonidus. AfO 22: 1-8.

Marzahn, J. and Schauerte, G. ed.

2008 Babylon. Wahrheit: eine Ausstellung des Vorderasiatischen Museums zu Berlin. München: Hirmer.

Maul, S. M.

1999 Der assyrische König-Hüter der Weltordnung. Pp. 201-214 in Priests and Officials in the Ancient Near East. Papers of the Second Colloquium on the Ancient Near East-The City and its Life, held at the Middle Eastern Culture Center in Japan (Mitaka, Tokyo), March 22-24, 1996. Edited by K. Watanabe. Heidelberg: Winter.

2012 a Tontafelabschriften des Kodex Hammurapi in altbabylonischer Monumentalschrift. ZA 102: 77-99.

2012 b Paläographische Übungen am Neujahrstag. Monumentalschrift. ZA 102: 202-208.

Mayer, W. R.

1987 Ein Mythos von der Erschaffung des Menschen und des Königs. OrNS 56: 55-68.

Sachs, A. J. and Hunger, H.

1988-2006 Astronomical Diaries and Related Texts from Babylonia. Wien: Verlag der Österreichischen Akademie der Wissenschaften.

Schaudig, H.

2001 Die Inschriften Nabonids von Babylon und Kyros' des Großen samt den in ihrem Umfeld entstandenen Tendenzinschriften. Textausgabe und Grammatik. AOAT 256. Münster: Ugarit-Verlag. 
Schnabel, P.

1923 Berossos und die babylonisch-hellenistische Literatur. Leipzig: Teubner.

Schroeder, O.

1922 Keilschrifttexte aus Assur historischen Inhalts. Zweites Heft. Leipzig: Hinrichs.

Soden, W. von

1932/33 Der Hymnisch-epische Dialekt. ZA 40 (1932): 163-227 and ZA 41 (1933): 90-183.

1995 Grundriss der akkadischen Grammatik. Analecta Orientalia 33. 3. Erg. Aufl. Roma: Pontificio Ist. Biblico.

Streck, M.

1916 Assurbanipal und die letzten assyrischen Könige bis zum Untergang Niniveh's. Leipzig: Hinrichs.

Wiseman, D. J. and Black, J. A.

1996 Literary Texts from the Temple of Nabû. Cuneiform Texts from Nimrud 4. London: British School of Archaeology in Iraq. 